Tropical Journal of Pharmaceutical Research February 2017; 16 (2): 313-318

ISSN: $1596-5996$ (print); 1596-9827 (electronic)

(C) Pharmacotherapy Group, Faculty of Pharmacy, University of Benin, Benin City, 300001 Nigeria.

All rights reserved.

Available online at http://www.tjpr.org

Original Research Article

http://dx.doi.org/10.4314/tjpr.v16i2.8

\title{
Preparation, characterization and antibacterial effects of eco-friendly gold nanorods
}

\author{
Manal A Awad ${ }^{1 *}$, Awatif A Hendi ${ }^{2}$, P Virk $^{3}$, Khalid MO Ortashi ${ }^{4}$, Mai A Elobeid ${ }^{3}$, \\ Nada M Merghani ${ }^{5}$, Muzzammil I Siddiqui ${ }^{3}$ and EM Ibrahim ${ }^{5}$ \\ ${ }^{1}$ King Abdullah Institute for Nanotechnology, ${ }^{2}$ Department of Physics, Faculty of Science, ${ }^{3}$ Department of Zoology, ${ }^{4}$ Department \\ of Chemical Engineering, ${ }^{5}$ Central Lab \& Prince Naif Centre for Heath Science Research, King Saud University, Saudi Arabia
}

*For correspondence: Email: a.manalawad@gmail.com, ahindi@ksu.esu.sa

Revised accepted: 14 January 2017

\begin{abstract}
Purpose: To synthesize and characterize eco-friendly gold nanorods (Au-NRs) and to assess their effects against two bacterial strains.

Methods: Synthesis of eco-friendly gold nanorods was done from an aqueous solution of chloroauric acid and cetyltrimethylammonium bromide by mixing Olea europaea fruit and Acacia nilotica husk extracts with the latter as a reducing agent. The synthesis was monitored by ultraviolet-visible (UV) spectrophotmetry and a zetasizer, while the morphology of the resulting nanorods was assessed by transmission electron microscopy (TEM) and scanning electron microscopy (SEM) coupled with an energy-dispersive spectrophotometer (EDS). The effect of the prepared eco-nanorods on growth of Escherichia coli and Staphylococcus aureus bacteria were investigated by agar diffusion method.

Results: The synthesized Au-NRs were mostly rod-shaped, with mean size of $96 \mathrm{~nm}$. They exhibited a potent antibacterial activity against Gram-positive and Gram-negative microorganisms (Escherichia coli and Staphylococcus aureus).

Conclusion: The findings suggest that the nanoformulation of the biomaterial mix of Olea europaea fruit and Acacia nilotica husk extracts is a cost-effective, eco-friendly, and non-toxic synthesis of $A u-$ NRs which has therpeautic potential.
\end{abstract}

Keywords: Olea europaea, Acacia nilotica, Gold nanorods, Eco-friendly synthesis, Gram-positive and Gram-negative microorganisms

Tropical Journal of Pharmaceutical Research is indexed by Science Citation Index (SciSearch), Scopus, International Pharmaceutical Abstract, Chemical Abstracts, Embase, Index Copernicus, EBSCO, African Index Medicus, JournalSeek, Journal Citation Reports/Science Edition, Directory of Open Access Journals (DOAJ), African Journal Online, Bioline International, Open-J-Gate and Pharmacy Abstracts

\section{INTRODUCTION}

Nanotechnology is a science that involves the manipulation of matter at the atomic and molecular levels. Nanobiotechnology is an integral and emerging tool for engineering ecofriendly nanoscale materials using natural biological sources [1]. The integration of inorganic nanoparticles (NPs) and biological structures is one of the most challenging areas of research. Biological synthesis elucidates the importance of metal-microbe interaction in several biotechnological applications, including bioremediation, biomineralization, bioleaching, and microbial corrosion [2].

NPs are unique in having a high surface area-tovolume ratio and high percentage of atoms at the grain boundaries [3,4]. Moreover, biological processes do occur at the nanoscale, which accounts for the ease of application of NPs in the field of medicine because of their amenability to biological functionalization [5]. NPs are broadly classified as organic and inorganic NPs. The 
latter have gained significant importance because of their ability to resist the processing conditions [6].

NPs are potent antibacterial and antifungal agents because of their ability to interact with microorganisms $[7,8]$. The antimicrobial activity of NPs is a function of the surface area in contact with the microbes. The small size and the high surface area-to-volume ratio (i.e., large surface area) of NPs helps them to interact well with microbes which affects their antimicrobial ability. The antimicrobial potential of metal-based NPs has led to their incorporation in consumer, health-related, and industrial products [9].

The synthesis and applications of stable gold nanoparticles (Au-NPs) have been a subject of great interest because of the remarkable physical and chemical properties of Au-NPs. Au-NPs are uniformly structured and are highly stable. In addition their size-related electronic, magnetic, and optical properties are judiciously exploited in fields such as catalysis, biosensing, drug delivery, energy-related applications, and biological applications $[10,11,12]$.

Metal NPs that exhibit antimicrobial activity when embedded and coated onto surfaces are widely used in water treatment, synthetic textiles, biomedical and surgical devices, and food processing and packaging [13]. Furthermore, antibiotics often disturb the bacterial flora of the digestive tract, leading to the development of multiple drug-resistant isolates; hence, novel methods for formulating biocide materials is imperative $[9,12,14,15]$. Thus, there is a need for a novel bactericidal that offers least resistance coupled with good bactericidal properties. AuNPs exhibit potent bactericidal effects on a wide range of microorganisms; however, the bactericidal effect is a function of the size and the shape of the structured nanoparticles [16].

With this premise, the present study aimed at the development of a novel method for the synthesis of gold nanorods (Au-NRs) by the reduction of aqueous $\mathrm{AuCl}_{4}$ and cetyltrimethylammonium bromide (CTAB), adopting an eco-friendly approach. The natural products used in the synthesis, we is a mix $(1: 3)$ of Olea europaea fruit extract and Acacia nilotica husk extract.

The antibacterial activity of Au-NRs against various strains of bacteria (Escherichia coli, Staphylococcus aureus) were tested. To our knowledge, method of preparation used in the study has not been reported in previous literature.

\section{EXPERIMENTAL}

\section{Reagents and chemicals}

For the synthesis of Au-NRs, all the chemicals used were of analytical grade. Chloroauric acid was purchased from Loba Chemie, India, and cetyltrimethylammonium bromide was purchased from BDH Laboratory supplies pool, BH151 TD, England. Olea europaea fruit and Acacia nilotica husk were obtained from Al-Jouf, Saudi Arabia and Sudan, respectively.

\section{Preparation of aqueous extract solution}

A $1 \%$ by volume $(\mathrm{g} / \mathrm{v})$ solution of Olea europaea fruit was washed well, ground in deionized water, and filtered. The extract was stored for later use. For Acacia nilotica husk extract, $1 \%$ by volume was soaked in deionized water overnight and filtered, and the extract was stored for later use. A mix of the $O$. europaea fruit extract and the $A$. nilotica husk extract with a volume ratio of $1: 3$ constituted the final organic solution. The solution contained flavonoids, phenols, and oleic acid and pentacyclic triterpenoid compounds, as revealed by thin-layer chromatography.

\section{Preparation of Au-NRs}

A novel approach was employed to synthesize eco-friendly Au-NRs. The nanorods (NRs) were synthesized by adding an aqueous solution comprising a mix $(1: 3)$ of $1 \%$ o europaea fruit extract and $1 \% A$. nilotica extract to an aqueous solution of $0.0009 \mathrm{~mol} / \mathrm{L}$ colloidal chloroauric acid $\left(\mathrm{HAuCl}_{4}\right)$ and $0.35 \mathrm{~mol} / \mathrm{L} \mathrm{CTAB}$ under vigorous stirring at a temperature of $45^{\circ} \mathrm{C}$ for 13 min until an orange color was observed in the mixture. When the extract was added, the mixture changed from orange to transparent and then became colorless. The colorless endpoint indicated the formation of NRs. The presence of Au-NRs were confirmed by the color changes and qualitatively characterized using an ultraviolet-visible (UV-vis) spectrophotometer. The microstructure was observed using a transmission electron microscope (TEM) and a scanning electron microscope (SEM).

\section{Instruments and apparatus}

The green synthesis of the Au-NRs was characterized spectrophotometrically. The UVvis spectra of the synthesized NRs were recorded with respect to time at room temperature using a UV-vis spectrometer (Perkin Elmer). The size of the synthesized AuNRs was determined using a Zetasizer system (Nano Series, HT Laser, ZEN3600, Malvern 
Instruments, UK). SEM was employed to characterize the shape and morphologies of the ecofriendly synthesized NRs (JEOL-FE-SEM), and an energy-dispersive X-ray spectroscopy (EDS) analysis was performed for the confirmation of elemental gold using (INCA x-act, Oxford Instruments) equipped for SEM. A transmission electron microscope (JEM-1011, JEOL, Japan) was employed to characterize the size, shape, and morphologies of the ecofriendly synthesized the Au-NRs.

\section{Antibacterial activity assay}

Pure cultures of Escherichia coli and Staphylococcus aureus bacteria were used. The antibacterial activity of the biosynthesized AuNRs were evaluated by the disk diffusion method. Nutrient agar medium plates were prepared, sterilized, and solidified. Thereafter, the bacterial cultures were swabbed on the plates. The sterile discs were dipped in a Au-NR solution (5 or $10 \mu \mathrm{g} / \mathrm{ml}$ ), placed on the nutrient agar plate, and incubated at $37^{\circ} \mathrm{C}$ for $24 \mathrm{~h}$. Upon inhibitory activity, a zone of clearing around the wells was observed. The diameter of the clearing zones was measured in millimeters using a ruler. The experiment was repeated three times, and the mean value of the zone diameter was recorded [17].

\section{RESULTS}

In our novel approach, green synthesis of AuNRs using CTAB as a surfactant and a mix (1:3) of Olea europaea fruit extract and Acacia nilotica husk extract was performed. The consistency of Au-NRs was successful, as indicated by the initial changes in color, and the Au-NRs exhibited a plasmon peak in the visible region (Figure 1).
An absorbance peak at $\sim 577 \mathrm{~nm}$ was observed in the spectrum.

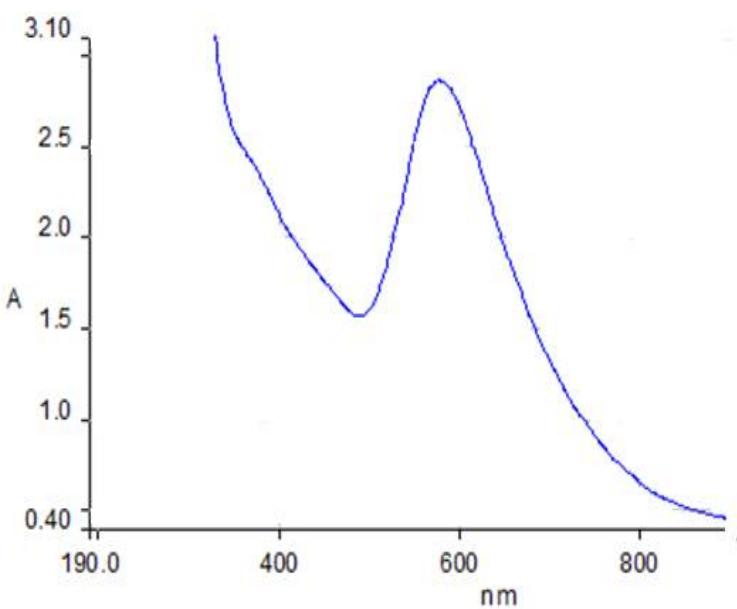

Figure 1: UV-vis spectra of Au-NRs synthesized with $0.09 \mathrm{~mol} / \mathrm{L} \mathrm{HAuCl}_{4}$

The mean size of the synthesized AuNRs was measured using a zetasizer system and was $\sim 96$ $\mathrm{nm}$, as shown in Figure 2.

TEM was used to determine the shape and size of the Au-NRs, as shown in Figures 3A, 3B, and 3C. Short nanorods are observed in Figure 3A, but long nanorods appear in Figures $3 \mathrm{~B}$ and $3 \mathrm{C}$.

Figure 4 shows the SEM image of the eco AuNRs synthesized by a mixture of Olea europaea fruit extract and Acacia nilotica husk extract, showing Au-NRs possessing rods shapes with monodispersity.

Figure 5 presents the EDS analysis for verifying the presence of gold in the suspension of NPs. Our results show a peak and confirm that gold was present in the suspension.

\section{Size Distribution by Intensity}

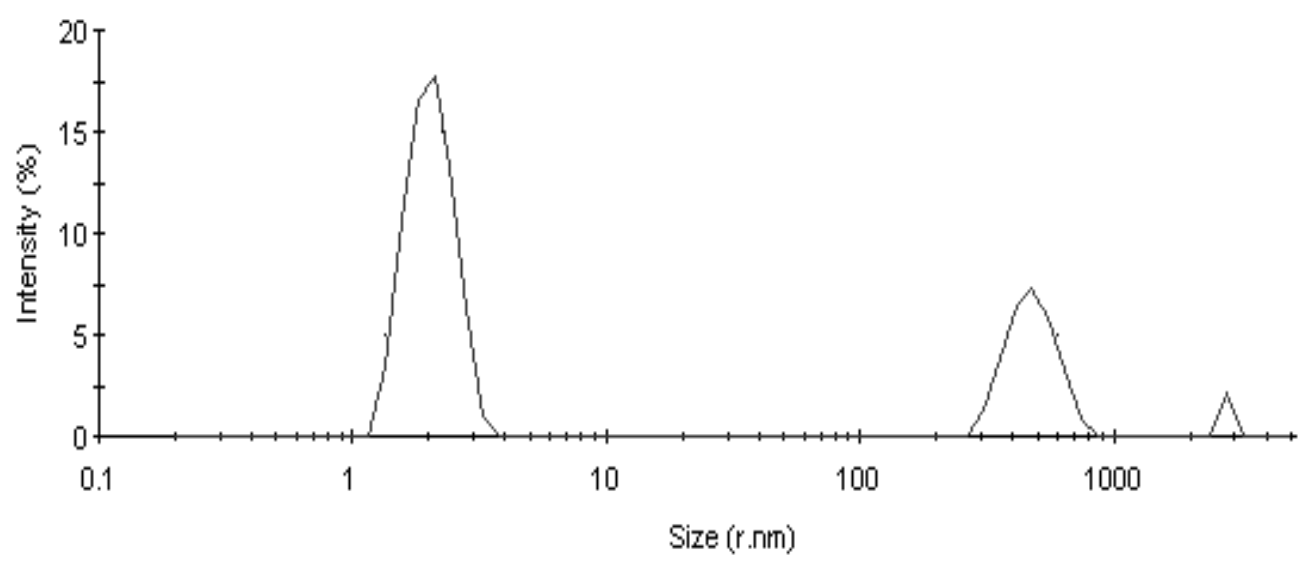

Figure 2: Zetasizer results for the nanoparticles 


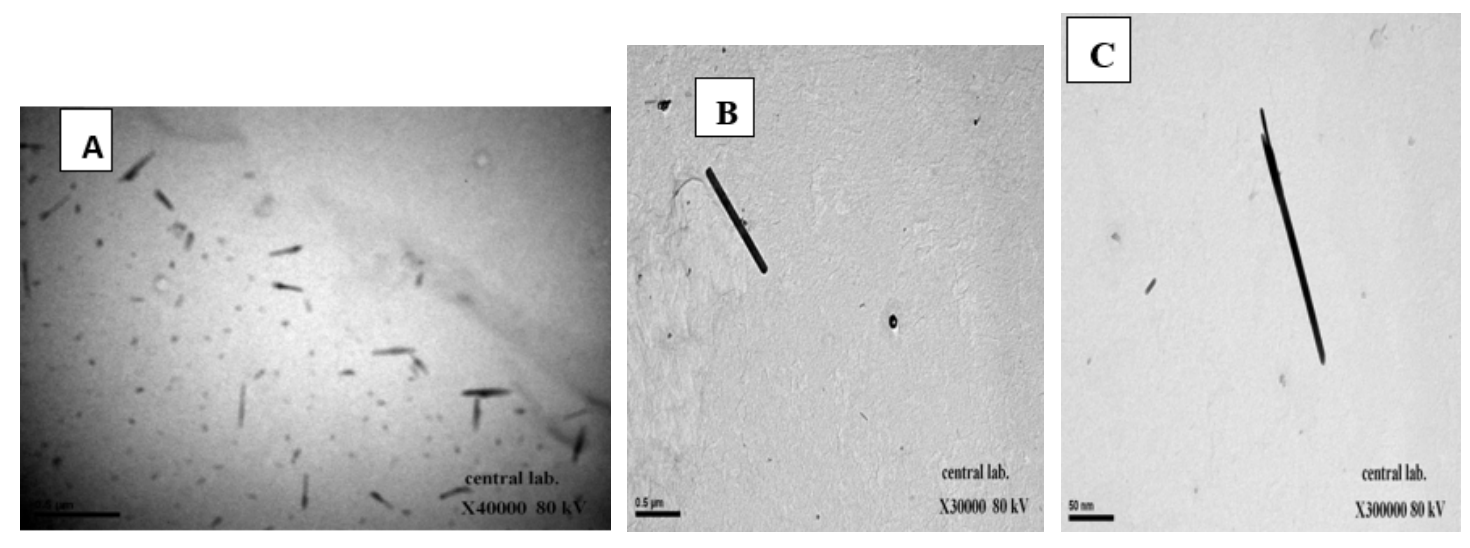

Figure 3: $(A, B, C)$ TEM images of the Au-NRs stabilized with the surfactant, CTAB, and a mix of Olea europaea fruit extract and Acacia nilotica extract

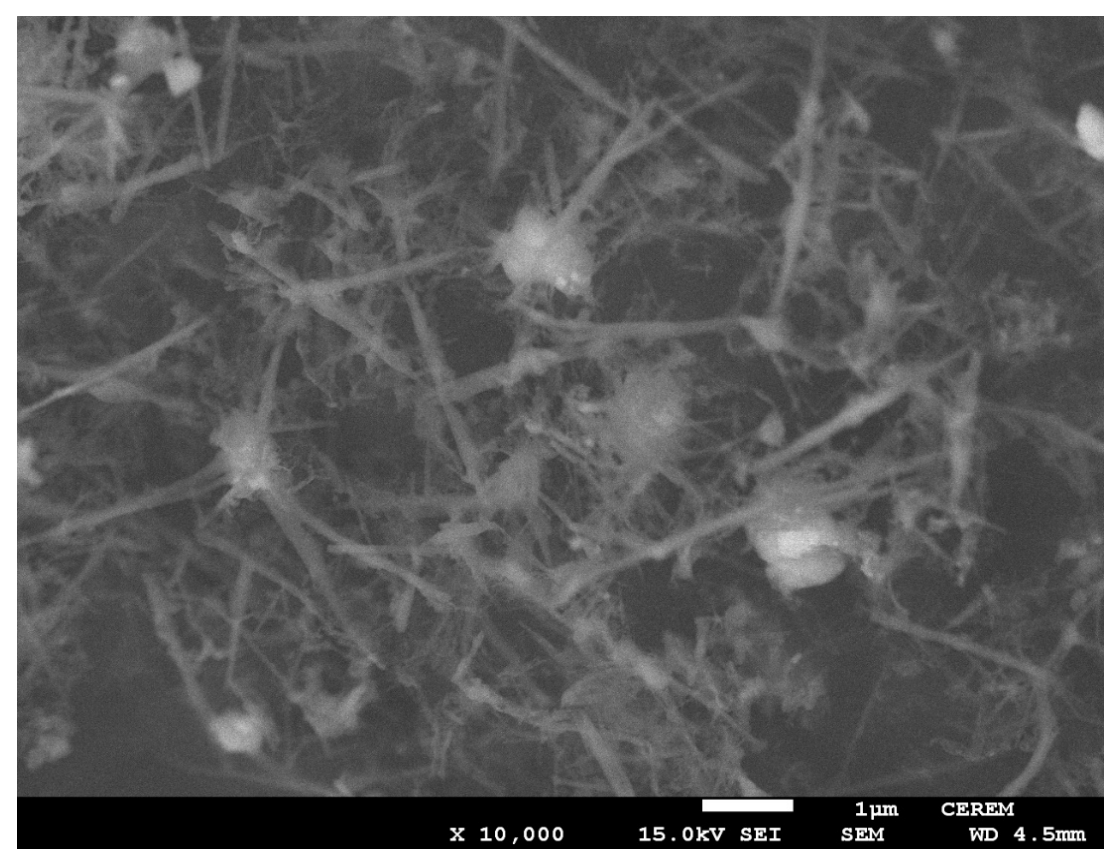

Figure 4: SEM image of Au-NRs synthesized by an aqueous mix of Olea europaea fruit extract and Acacia nilotica husk extract

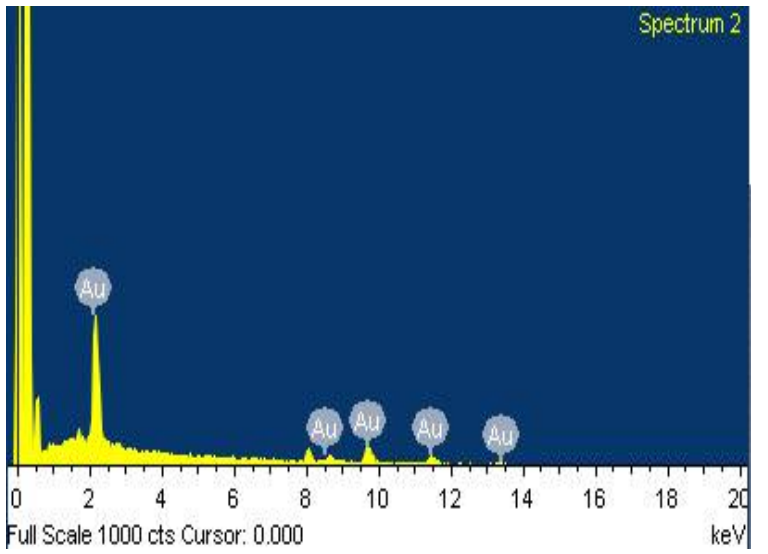

Figure 5: EDS results showing the content (\%) of elemental gold in the resulting suspension and the spectrum of the Au-NRs

\section{Antibacterial activity}

Using our novel approach, we determined the antibacterial activity of eco-friendly Au-NRs with an average size of $96 \mathrm{~nm}$ at different concentrations (5 or $10 \mu \mathrm{g} / \mathrm{ml}$ ) via agar platebased growth studies on Gram-positive and Gram-negative microorganisms (Escherichia coli, Staphylococcus aureus). The results of the bacterial growth studies showed that the Au-NRs at both varying concentrations $(5,10 \mu \mathrm{g} / \mathrm{ml})$ exhibited a significantly higher toxicity against Staphylococcus aureus in comparison to the Escherichia coli. This was evident from the diameter of the zone of inhibition which was $24 \mathrm{~mm}$ and $19 \mathrm{~mm}$ for $S$. aureus at 10 and 5 $\mu \mathrm{g} / \mathrm{ml}$, respectively, while for $E$. coli, the diameter 
of the zone of inhibition was 16 and $15 \mathrm{~mm}$ at 10 and $5 \mu \mathrm{g} / \mathrm{ml}$, respectively.

\section{DISCUSSION}

The mechanism of formation of rod-shaped NPs in aqueous surfactant media remains unclear. In the present study, a novel ecofriendly synthesis of Au-NRs was successfully performed. The consistency of Au-NRs was evident, by the initial changes in color, and the Au-NRs exhibited a plasmon peak in the visible region. The formation of Au-NRs was confirmed by recording the UVvis spectrum of the colloidal solution. An absorbance peak at $\sim 577 \mathrm{~nm}$ was observed in the spectrum. The sharpness of the peak indicated that the nanorods were monodispersed.

The average size of the synthesized AuNRs was measured using a zetasizer system and was $~ 96$ $\mathrm{nm}$ with monodispersity. These results of the Zetasizer clearly indicated that the particles were fairly stable with uniform sizes, this was in line with the results of the UV-vis spectrum.

The shape and size distribution for the stable AuNRs were characterized by particle characterization and TEM analysis. Short and long nanorods appear in Figures $3 \mathrm{~B}$ and $3 \mathrm{C}$. Our results are similar to those of another study performed on long NRs prepared in the absence of silver nitrate $[18,19]$. The TEM images show that $\mathrm{Au}-\mathrm{NPs}$ have various sizes, which agrees with the results obtained from the analysis of the UV-vis spectrum and the Zetasizer readings.

SEM images exhibited Au-NRs were rod shaped with monodispersity, and EDS analysis confirmed the presence of gold in the suspension.

Gold particles in particular extensively exploited in biology owing to their biocompatibility [19]. AuNPs are generally considered to be biologically inert but can be structured to possess chemical or photothermal functionality. Under near-infrared (NIR) irradiation, gold-based nanomaterials, nanospheres, nanocages, and Au-NRs with characteristic NIR absorption can destroy cancer cells and bacteria via photothermal heating. AuNRs conjugated with photosensitizers can kill methicillin-resistant Staphylococcus aureus via photodynamic antimicrobial chemotherapy and NIR photothermal radiation $[20,21]$.

Using the novel approach of the present study, we evaluated the antibacterial activity of ecofriendly Au-NRs, with an average size of $96 \mathrm{~nm}$, at different concentrations (5 and $10 \mu \mathrm{g} / \mathrm{ml}$ ) via agar plate-based growth studies on Grampositive and Gram-negative microorganisms (Escherichia coli, Staphylococcus aureus). The resulting Au-NRs at both concentrations exhibited a very high toxicity against $S$. aureus than E. coli. Thus the study reports a successful ecofriendly synthesis of Au-NRs with antibacterial activity against both Gram-positive and Gram-negative bacteria hence, Au-NRs synthesized by the proposed method could prove to be effective in antimicrobial applications. The biological method employed saves a large amount of energy and is eco-friendly, and economical. Moreover, it does not involve the use of any toxic chemicals.

\section{CONCLUSION}

The key findings of the present study include an eco-friendly synthesis of Au-NRs using CTAB as a surfactant and a mixture (1:3) of Olea europaea fruit extract and Acacia nilotica husk extract. can be achieved, The technique used is also, simple and cost-effective, and does not involve the use of toxic chemicals. Olea europaea fruit extract and Acacia nilotica husk extract play an important role in the reduction and stabilization of gold. The method employed in this study synthesis may also be suitable for the preparation of nanomaterials for use in the pharmaceutical and biomedical fields as well as in other industries.

\section{DECLARATIONS}

\section{Acknowledgement}

The authors extend their appreciation to the Deanship of Scientific Research at King Saud University for funding this work through Research Group no. RGP- 278.

\section{Conflict of Interest}

No conflict of interest associated with this work.

\section{Contribution of Authors}

The authors declare that this work was done by the authors named in this article and all liabilities pertaining to claims relating to the content of this article will be borne by them.

\section{Open Access}

This is an Open Access article that uses a funding model which does not charge readers or their institutions for access and distributed under the terms of the Creative Commons Attribution 
License (http://creativecommons.org/licenses/by 14.0) and the Budapest Open Access Initiative (http://www.budapestopenaccessinitiative.org/rea d), which permit unrestricted use, distribution, and reproduction in any medium, provided the original work is properly credited.

\section{REFERENCES}

1. Gilaki M. Biosynthesis of silver nanoparticles using plant extracts. J. Biol. Sci. 2010; 10: 465-467.

2. Singh M, Manikandan S, Kumaraguru AK. Nanoparticles: A new technology with wide applications. Res. J. Nanosci. Nano. Technol. 2011; 1: 1-11.

3. Daniel MC, Astruc D. Gold nanoparticles: assembly, supramolecular chemistry, quantum-size-related properties, and applications toward biology, catalysis, and nanotechnology. Chem. Rev. 2004; 104: 293-346.

4. Kato $H$. In vitro assays: tracking nanoparticles inside cells. Nat. Nanotechnol. 2011; 6: 139-140.

5. Parak WJ, Gerion D, Pellegrino T, Zanchet D, Micheel C, Williams CS, Boudreau R, Le Gros MA, Larabell CA, Alivisatos AP. Biological applications of colloidal nanocrystals. Nanotechnology 2003; 14: 15-27.

6. Whitesides GM. The 'right' size in nanobiotechnoloy. Nature Biotechnol. 2003; 21: 1161-1165.

7. Mohanpuria P, Rana NK, Yadav SK. Biosynthesis of nanoparticles: technological concepts and future application. J. Nanopart. Res. 2008; 10: 507-517.

8. Dushenkov V, Kumar PB, Motto H, Raskin I, Rhizofiltration: The use of plants to remove heavy metals from aqueous streams. Environ. Sci. Technol. 1995; 29: 1239-1245.

9. Dibrov P, Dzioba J, Gosink KK, Hase CC. Chemiosmotic mechanism of antimicrobial activity of $\mathrm{Ag}+$ in Vibrio cholera. Antimicrob. Agents. Chemother. 2002; 46 . 2668-2670.

10. Ashok Kumar S, Chang Y-T, Wanga S-F, Lu H-C. Synthetic antibacterial agent assisted synthesis of gold nanoparticles: Characterization and application studies. J. Phys. Chem. Solids 2010; 71: 1484-1490.
11. Liu J, Qiao SZ, Lu GQ. Magnetic nanocomposites with mesoporous structures: synthesis and application. Small 2011; 7: 425-443.

12. Han G, Ghosh PM, Rotello VM. Drug and Gene Delivery using Gold Nanoparticles. J. Nanobiotech. 2007; 3: 4045.

13. Gutierrez FM, Olive PL, Banuelos A, Orrantia E, Nino N, Sanchez EM, Ruiz F, Bach H, Gay YA. Synthesis, characterization, and evaluation of antimicrobial and cytotoxic effect of silver and titanium nanoparticle. Nanomedicine 2010; 6: 681-688.

14. Daglia M, Papetti A, Grisoli P, Aceti C, Dacarro C, Gazzani G. Antibacterial activity of red and white wine against oral streptococci. J. Agric. Food Chem. 2007; 55: 5038-5042.

15. Jarvinen H, Tenovuo J, Huovinen P. In vitro susceptibility of Streptococcus mutans to chlorhexidine and six other antimicrobial agents. Antimicrob. Agents. Chemother. 1993; 37: 1158-1159.

16. Nirmala GA, Pandian K. Antibacterial efficacy of aminoglycosidic antibiotics protected gold nanoparticlesA brief study, Colloid Surface A 2007; 297: 63-70.

17. Savithramma N, Linga Rao M, Rukmini $K$, Suvarnalatha $P$. Antimicrobial activity of silver nanoparticles synthesized by using medicinal plants. Int J ChemTech Res 2011; 3: 1394-1402.

18. Jana NR, Gearheart L, Murphy CJ. Wet chemical synthesis of high aspect ratio cylindrical gold nanorods. J. Phys. Chem. B 2001; 105: 4065-4067.

19. Bhattacharya R, Mukherjee P. Biological properties of "naked" metal nanoparticles. Adv. Drug Deliv. Rev. 2008; 60: 1289-1306.

20. Ryan BM, Dougherty TJ, Beaulieu D, Chuang J, Dougherty BA, Barrett JF. Efflux in bacteria: what do we really know about it? Expert Opin. Investig. Drugs 2001; 10: 1409-1422.

21. Zharov VP, Mercer KE, Galitovskaya EN, Smeltzery MS. Photothermal nano therapeutics and nanodiagnostics for selective killing of bacteria targeted with gold nanoparticle. Biophys. J. 2006; 90: 619-627. 